\title{
Effect of chain topology on plasmonic properties of pressure sensor films based on poly(acrylamide) and Au nanoparticles
}

\author{
Gokhan Topcu, Mustafa M. Demir* \\ Department of Materials Science and Engineering, Izmir Institute of Technology, 35430, Gülbahçe, Urla, Izmir, Turkey
}

\section{A R T I C L E I N F O}

\section{Article history:}

Received 1 April 2019

Received in revised form 1 May 2019

Accepted 6 June 2019

Available online 7 June 2019

\section{Keywords:}

Crosslinking

Disassembly

Gold nanoparticle

Plasmonic shift

Pressure sensor

\begin{abstract}
A B S T R A C T
Au nanoparticles have been recognized as a colorimetric sensing element in polymeric systems because clustering shifts the red color of individual particles into saturated blue due to distinct plasmonic variation. The mechanism of pressure sensing is based on the disintegration of the particle clusters into the individual particles in polymers upon application of pressure. Polymers are usually composed of linear chains that provide a viscoelastic medium for their diffusion. Changing topology of polymer chains from linear to crosslinked under fixed pressure makes a clear change in spectral features of the particles probably due to the hindrance of particle diffusion by the crosslinking points. Therefore, the working range of the sensor films can be increased to higher-pressure values. In this work, polyacrylamide/Au nanoparticle films were prepared by various concentrations of formaldehyde as a crosslinking agent from 0.5 to $5.0 \mathrm{wt}$ \%. The initial absorption signal gradually shifts from 690 to $545 \mathrm{~nm}$ for linear chains upon application of pressure while shifting goes down to $571 \mathrm{~nm}$ for crosslinked ones. The colorimetric change is also examined under humid environments. Contrary to the crosslinking process, humid environment facilitates the diffusion of particles since the chains swell with water molecules that provide a convenient medium for particle diffusion.
\end{abstract}

(c) 2019 Elsevier B.V. All rights reserved.

\section{Introduction}

Pressure sensors have attracted great attention over the past decade due to their potential usage in various applications including object detection, haptic displays, and fingerprint sensors, etc. [1-3]. Pressure-sensing elements have been categorized into three types according to their piezoresistive [4-8], capacitive [9-11], or piezoelectric $[12,13]$ features. However, the colorimetric pressure sensitive films have emerged as a new class of sensor that offers not only recognizes the amplitude of applied force but also characterizes stress distribution. Since the accurate real-time detection of the change in pressure loading is vital for the construction and industrial fields, the use of colorimetric pressure elements in a static body is important for the prediction of catastrophic failures [14].

Colorimetric pressure sensitive films can be obtained using colloidal nanoparticles (NPs) to take the advantage of optical properties of their assembly [15]. In addition to their size- and dimensional-dependent outcomes, the colloidal nanoparticles can exhibit outstanding optical features due to a collective behaviour, namely interparticle coupling [16]. For instance, gold nanoparticles

\footnotetext{
* Corresponding author.

E-mail address: mdemir@iyte.edu.tr (M.M. Demir).
}

are the sine qua non to fabricate stimuli-responsive smart materials as a sensor due to their distinct plasmonic properties both individually and collectively. Under an incident visible light, their free surface electrons are confined and can absorb the part of light having a specific wavelength. This phenomenon defined as localized surface resonance (LSPR) and can be tuned by the carrier of the particles [17]. Since the control over LSPR by assembly or disassembly of the nanoparticles leads to a saturated change in color, the Au NP-based colorimetric sensors have been developed for a wide variety of functions including $\mathrm{pH}$ [18-20], temperature [21,22], biochemical sensing [23], and strain [24,25]. Moreover, the repealing of stimulus may reverse the color to the initial state. Hence, colorimetric sensors consisting of plasmonic nanoparticles offer both reusability and tailorable specificity for the measurement.

The particles can be associated with the surrounding polymer matrix whose mechanical and viscoelastic properties play a significant role. For instance, Han et al proposed that the addition of poly(ethylene glycol) into poly(vinyl pyrrolidone) (PVP) as a plasticizer remarkably facilitate and enhance the plasmonic shift due to provide ease of diffusion of Au NPs, therefore their separation [24]. In addition, the disassembly of plasmonic nanoparticles upon external mechanical stimuli is more favorable if the polymer has pendant group that is able to easily interact with the particle, e.g. pyrrolidone or amide [26]. Since the surrounding matrix 


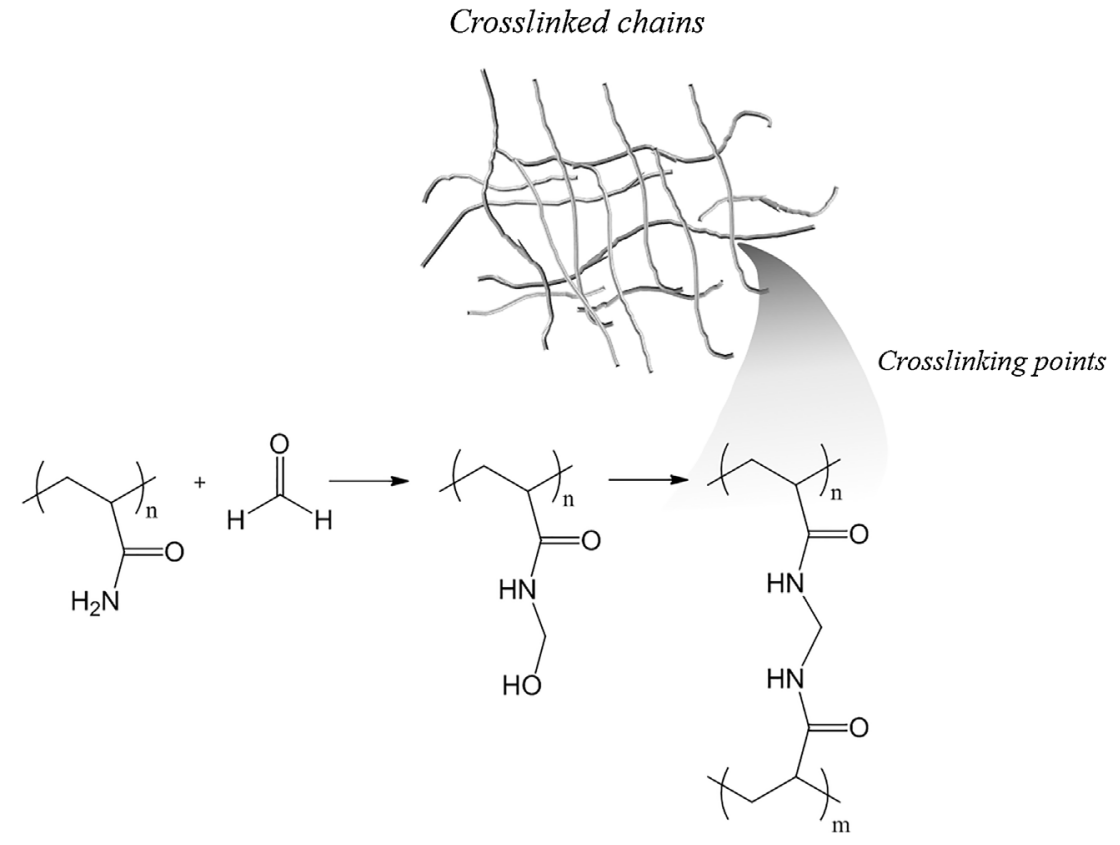

Fig. 1. Schematic illustration of the crosslinking reaction.

plays a vital role for the disassembly of the nanoparticles, hindering particle diffusion through various topologies of polymer chains may offer an alternative for the fabrication of colorimetric pressure sensors working at higher-pressure ranges in various fields such as industrial machinery, construction, agriculture, where highpressure monitoring is essential.

The polymers consisting of linear chains intrinsically resemble liquids with regard to their flow behavior. On the contrary, the crosslinked chains provide solidification and maintaining the material shape against deformation. Therefore, the crosslinking of polymer chains has been predominantly used for the enhancement of the material toughness and chemical inertness due to its ease of implementation. A three-dimensional network of polyacrylamide (PAAm) can be obtained by using aldehyde compounds due to the capability of reacting with amide groups [27-29].

This study provides insight into the effect of polymer chain topology on the optical properties of Au NP/PAAm films upon application of pressure. The fabrication of the colorimetric pressure sensor films was carried out by integration of Au NPs into PAAm matrix. The spectral changes by disassembly of Au NP clusters were observed as a function of the amount of crosslinking agent (formaldehyde) and the applied pressure. Besides crosslinking of the PAAm chains, the humidity was also studied since the motion of the particles in the polymeric system is significantly influenced. The effect of humidity on colorimetric response against pressure and color healing is examined.

\section{Experimental}

\subsection{Materials}

Tetrachloroauric (III) acid trihydrate $\left(\mathrm{HAuCl}_{4} \cdot 3 \mathrm{H}_{2} \mathrm{O}, 99 \%\right.$, SigmaAldrich), trisodium citrate dihydrate ( $\geq 99 \%$, Sigma-Aldrich), and PVP $\left(\mathrm{M}_{\mathrm{W}}: 10 \mathrm{~kg} / \mathrm{mol}\right.$, Sigma-Aldrich) were used to Au NP synthesis. PAAm solution ( $50 \% \mathrm{w} / \mathrm{v}, \mathrm{M}_{\mathrm{w}}: 1500 \mathrm{~g} / \mathrm{mol}$, Sigma-Aldrich), methanol ( $\geq 99.8 \%, \mathrm{VWR})$ and formalin $(40 \% \mathrm{w} / \mathrm{v}$ formaldehyde, Carlo Erba) were purchased and used to prepare polymer network having various concentration of crosslink agents. The deionized water $\left(18.2 \mathrm{M} \Omega \cdot \mathrm{cm}^{-1}\right.$ at $\left.25^{\circ} \mathrm{C}\right)$ used in all experiments was produced by a Milli-Q Advantage water treatment system. All chemicals were used as received without any further purification.

\subsection{Synthesis of Au NPs}

The synthesis of Au NPs was performed according to Turkevich method via reduction of $\mathrm{HAuCl}_{4}$. An aliquot of aqueous solution of $\mathrm{HAuCl}_{4}(0.24 \mathrm{mM}, 200 \mathrm{~mL})$ was loaded to a glass container and heated up until reflux with magnetic stirring. Afterward, trisodium citrate solution $(0.34 \mathrm{M}, 1 \mathrm{~mL})$ was rapidly injected to the system. The solution was allowed to reflux for $30 \mathrm{~min}$ while its color changed from pale yellow to dark blue and finally ruby red. The resulting dispersion was centrifuged $(12,000 \mathrm{rpm}, 15 \mathrm{~min})$ and redispersed in $2 \mathrm{~mL}$ water.

\subsection{Synthesis of crosslinked PAAm}

The crosslinking was obtained by the reaction between PAAm and formaldehyde (FA) (Fig. 1). An aliquot of PAAm solution ( $2 \mathrm{~mL}$ ) was diluted to $35 \% \mathrm{w} / \mathrm{v}$ using $0.84 \mathrm{~mL}$ deionized water. Subsequently, the mixture was acidified using $25 \mu \mathrm{L} \mathrm{HCl}$ solution $(0.1 \mathrm{M})$ and loaded to a sealed glass container to purge with nitrogen. The desired amount of formalin solution was (from 0.5 to $5 \% \mathrm{~mol}$ of PAAm) injected to the system. The solution was then allowed for mixing at $60^{\circ} \mathrm{C}$ for $12 \mathrm{~h}$. The resulting polymers were precipitated using an excess amount of methanol. Afterward, the white precipitates were filtered and washed with methanol 3 times and dried at $40^{\circ} \mathrm{C}$ under vacuum. The dried white powders were dissolved in deionized water and the concentration was fixed to $50 \% \mathrm{w} / \mathrm{v}$ for further use.

\subsection{Preparation of pressure sensor films}

Firstly, Au NP $(50 \mu \mathrm{L})$ solution was mixed to PVP solution $(10 \mu \mathrm{L}$, $0.16 \% \mathrm{w} / \mathrm{v}$ in water) to enhance the stabilization of the particles. Next, the PVP modified Au NPs were loaded to PAAm solutions $(400 \mu \mathrm{L})$, respectively. The solution of which the ruby red color rapidly transformed into deep blue color was drop-casted into a mold and dried at $60^{\circ} \mathrm{C}$ for $2 \mathrm{~h}$. 

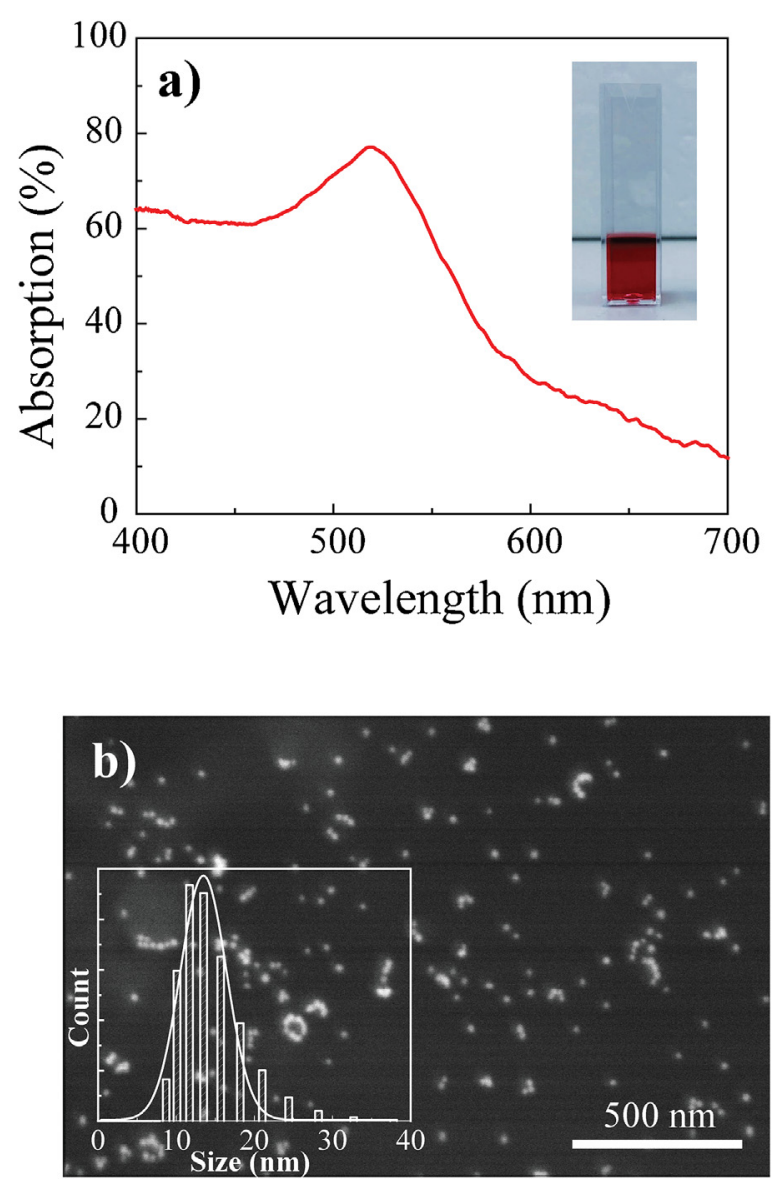

Fig. 2. Detailed approach for Au NPs; (a) absorption spectrum (inset shows appearance of dispersion), (b) SEM image (inset shows DLS size distribution).

\subsection{Characterization}

The resulting composite films were cut into small pieces with $1 \mathrm{~mm}$ diameter and were placed into a hydraulic pressure apparatus (Carver Inc., Model \#3925, Wabash, IN, USA). Constant and uniaxial pressures (from $40 \mathrm{MPa}$ to $120 \mathrm{MPa}$ ) were perpendicularly applied onto film surface for $2 \mathrm{~min}$ at room temperature. The size distribution of Au NPs was measured using Dynamic Light Scattering (DLS; Malvern, Zetasizer ZS90, UK). The morphology of the particles was studied by Scanning Electron Microscopy (SEM; Quanta 250, FEI, Hillsboro, OR, USA). Optical properties of compressed and non-compressed samples were recorded in absorbance mode using USB2000+ spectrometer (Ocean Optics Inc., Dunedin, FL, USA) via a premium fiber cable. The photographs of the films were taken with a cell phone camera (Apple, Iphone 7, CA, USA).

\section{Results and discussion}

$\mathrm{Au}$ NPs were obtained by reduction of $\mathrm{Au}^{3+}$ to $\mathrm{Au}^{\circ}$ by citrate molecules, which also act as a stabilizer. Since the coloring of the Au system requires meticulous control over the aggregation behavior of the nanoparticles, it is vital to study the stabilization of the particles by PVP. The photo of the Au NP dispersion and corresponding UV-vis absorption spectrum is given Fig. 2a. The single and sharp signal is observed at $521 \mathrm{~nm}$. The particle morphology was studied by SEM and corresponding microscopy image is given in Fig. $2 \mathrm{~b}$. The particles show a spherical shape and approximately $14 \mathrm{~nm}$ with diameter. Size distribution of the total population of the particles is presented as an inset of the figure. The diameter of the particles found as $13.5 \mathrm{~nm}$ and exhibit Gaussian distribution through
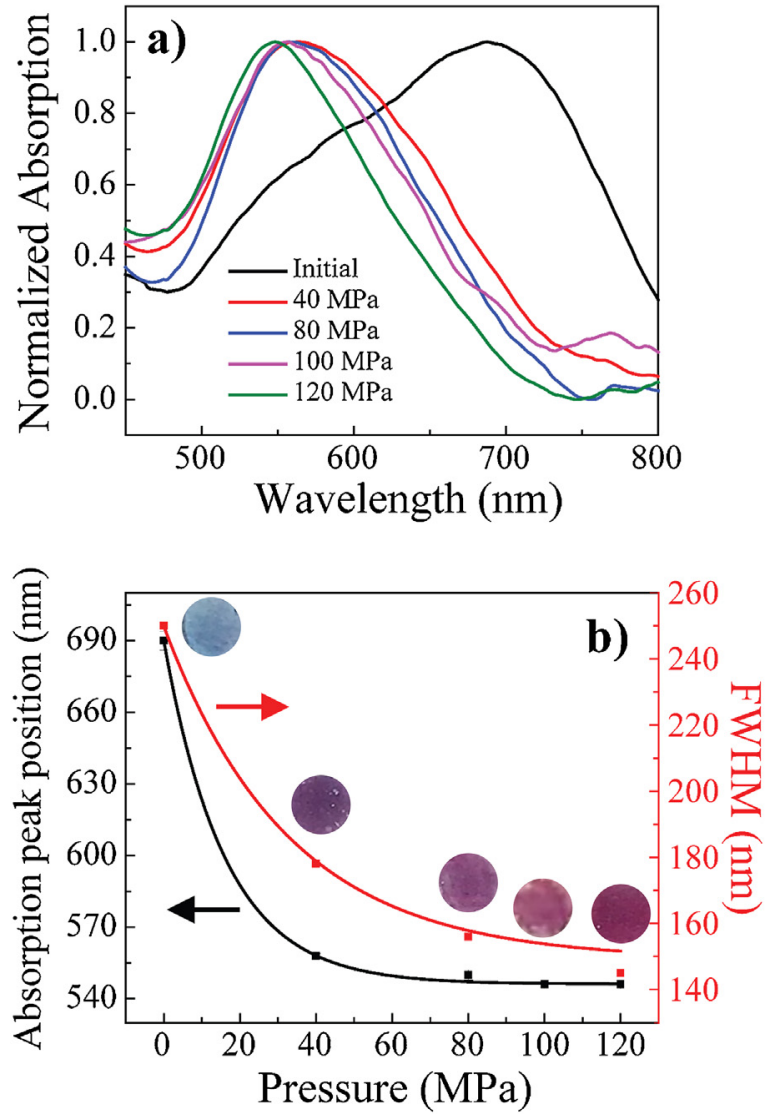

Fig. 3. (a) Absorption spectra and corresponding (b) peak position and FWHM of neat PAAm/Au film under various pressure at $73 \% \mathrm{RH}$. Insets show appearance of film color.

light scattering measurement, which is consistent with the results of the microscopy images.

The addition of PAAm solution into PVP-stabilized Au NP dispersion causes aggregation probably due to the change in $\mathrm{pH}$. The detachment of remaining citrate molecules from the particle surface may lead to aggregation of the particles into clusters. Therefore, the color of the mixture changes from red to blue. By evaporation of the water from the mixture, the blue appearance was fixed and the composite films were obtained. The blue films were divided into small pieces to perform compression experiments between 40 and $120 \mathrm{MPa}$. The intensive compression onto PAAm/Au films resulted in a significant color change from blue to red. The corresponding absorption spectra of the film for each pressure are given in Fig. 3a. For initial state, the broad signal is observed at $690 \mathrm{~nm}$ with a shoulder. The position of the shoulder $(550 \mathrm{~nm})$ is close to the signal of the individual particle dispersion. There is a clear difference between the absorption signal of individual Au particles $(520 \mathrm{~nm})$ and its aggregates $(690 \mathrm{~nm})$. Since these two levels of particle state coexist in the composite system, the effect of pressure is mainly examined by their spectroscopic signals. For this particular spectrum, it can be stated that the system shows bimodal distribution [30]. In other words, a few numbers of individual particles still exist together with aggregates in the dried form of the blue composite. As the pressure increases, the absorption spectrum clearly shows a shift toward lower wavelength and finally reaches to $545 \mathrm{~nm}$ for $120 \mathrm{MPa}$ compression. This dramatic spectral change is originated from the pressure-induced disassembly of Au NP aggregates. Nevertheless, the difference between peak positions is nearly $25 \mathrm{~nm}$ obtained from individual particle dispersion and compressed films. This result suggests that complete disassembly cannot be achieved 

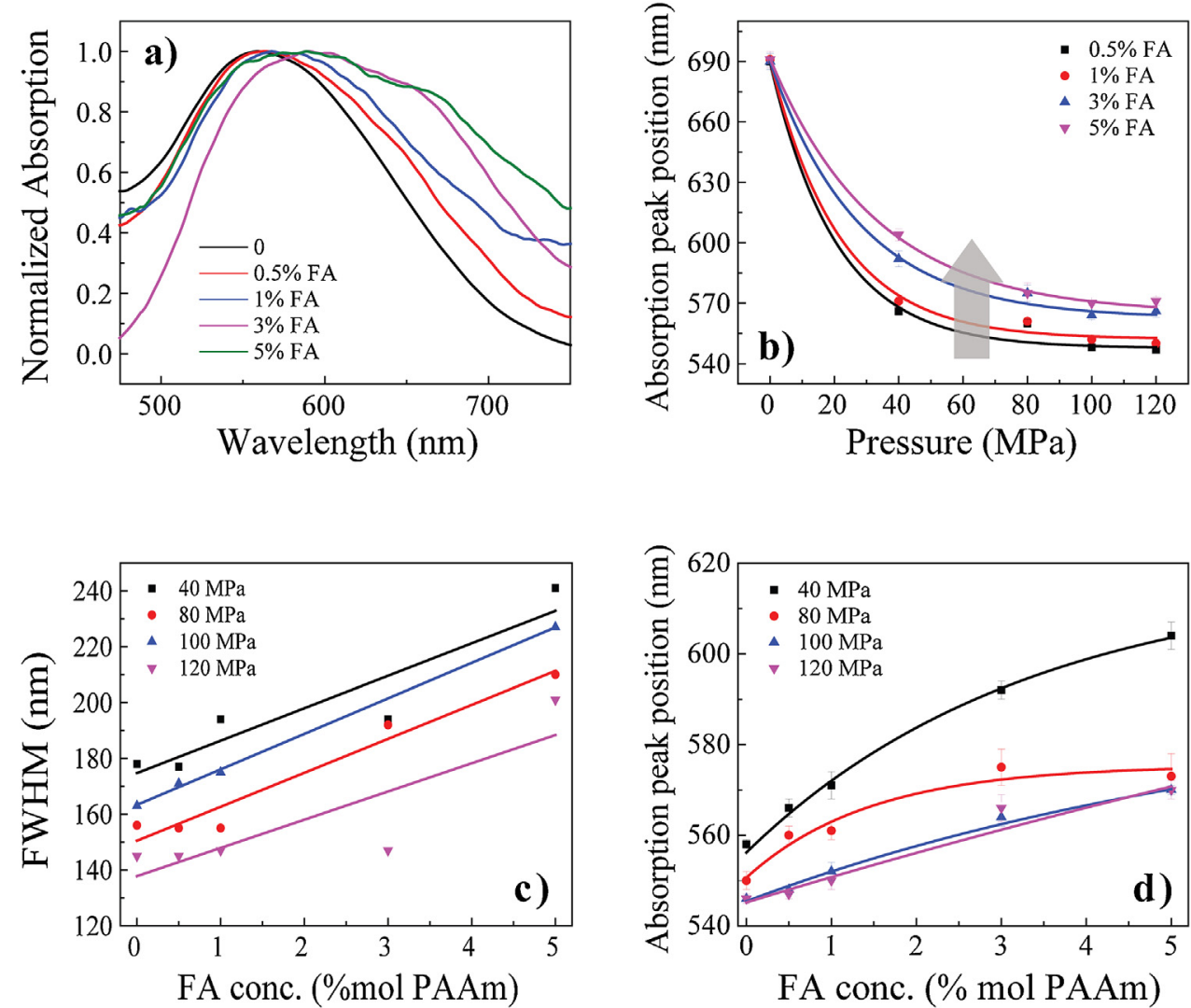

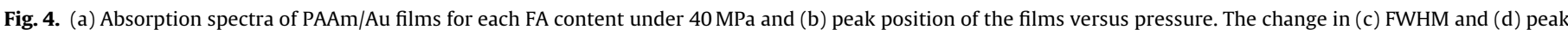
position of the films with changing FA content, respectively. The measurements were carried out at $73 \% \mathrm{RH}$.
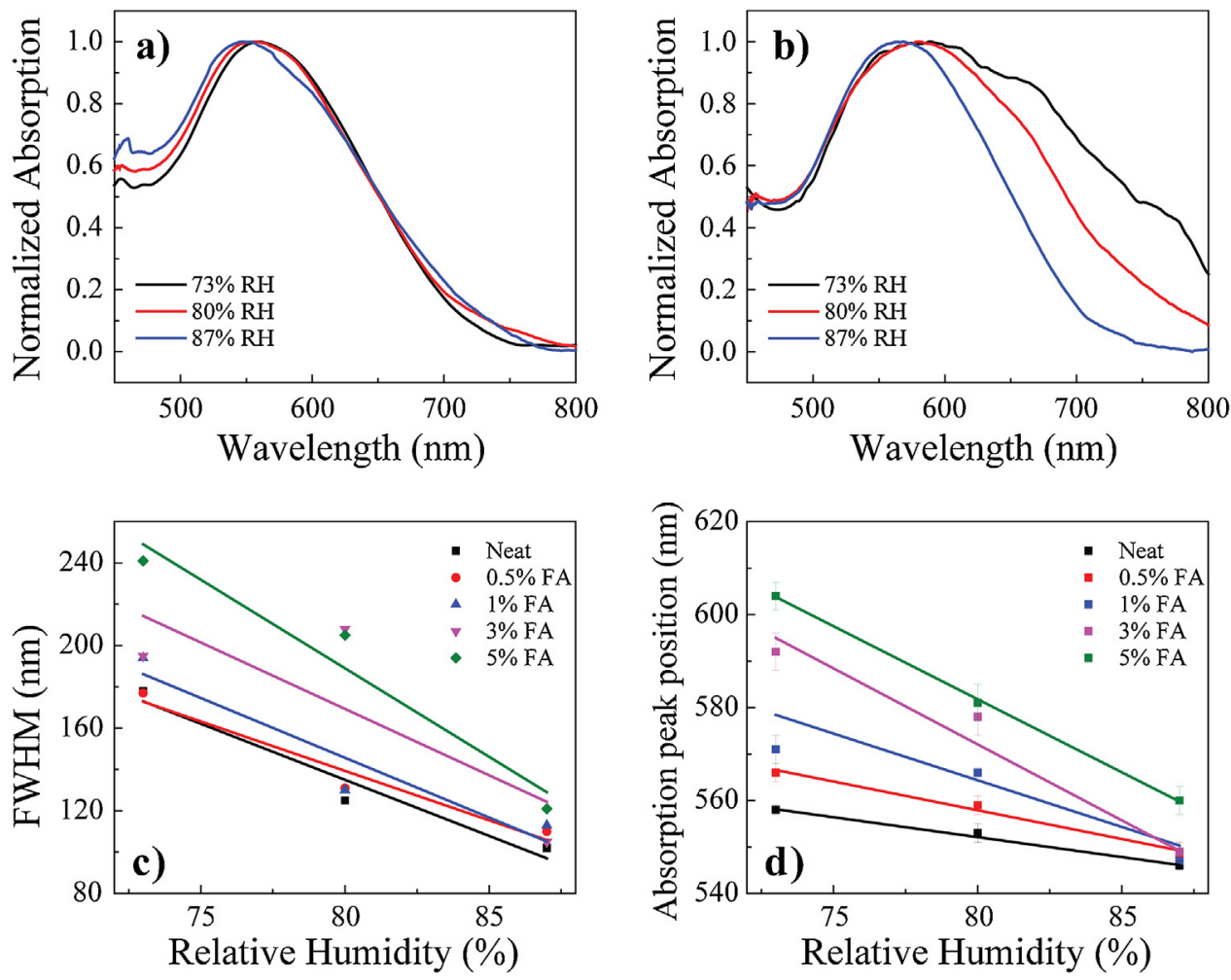

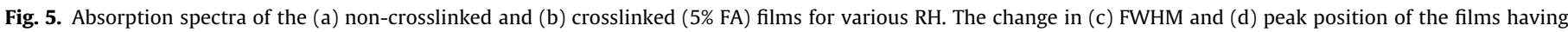
various FA content with changing RH, respectively. The measurements were carried out under $40 \mathrm{MPa}$. 
even at higher pressure; rather aggregated domains still exist in the compressed film structure. Besides the spectral shift, the shape of the spectra also changes. The absorption signals get narrow as the pressure increases. Both dissipative and radiative mechanisms to the damping of the collective electron oscillation may contribute to this result [31-33]. More specifically, light re-emission (scattering) becomes negligible for small spherical metal nanoparticles, which leads to the spectral narrowing of the LSPR with disassembly of aggregates. The limit of spectral narrowing may be result of the dissipative mechanisms. The reduction of the collective charge oscillation into single electron-hole pairs in the different (interband) or same (intraband) bands, namely Landau damping, affects the intrinsic optical properties of the nanoparticles [34]. In Fig. 3b, the changes (shift and narrowing) in the spectra with respect to pressure are given. Both the signal position and full width at half maximum (FWHM) of the spectrum show an exponential decrease as the pressure increases. On the other hand, the appearance of films for each compression level is presented as an inset of the figure such that a clear change in color from blue to red is clearly observed.

The spectral change at constant pressure was examined as a function of FA content used during the preparation of the films. The absorption spectrum of each film having different FA content under $40 \mathrm{MPa}$ compression is presented in Fig. 4a. The signal position of the non-crosslinked film in the spectrum falls to $556 \mathrm{~nm}$ while it shifts to higher wavelength by increasing FA concentration. Moreover, the signal is getting broaden at longer wavelengths. For the disassembly of the particles in the polymer matrix, the general sense is the use of the polymer that can allow continuous flow under the external mechanical stimuli. On the other hand, the use of FA leads to the reduction in the flow rate of the matrix under fixed mechanical force. The effect of crosslinking on the optical properties of the film has been investigated for the structural development of PAAm/Au particle nanocomposite materials. It was previously reported that the crosslinking points of the polymer chains reduce the free volume in the material, hence hinder the diffusion of the nanoparticles [35,36]. Therefore, the Au NP aggregates with an inhibited diffusion result in relatively weaker disassembly under similar mechanical stimuli compared to the particles in a non-crosslinked matrix. Thus, the shift in absorbance decreases as the concentration of crosslinking agent increases. The higher crosslinking, the harder the particles diffuse. In Fig. 4b, the change in peak position with respect to pressure for different FA content is presented. Obviously, all regimes follow a dramatic decrease as the pressure increases and the range of shift differs for each film. At high concentration of the crosslinking agent, this range gets narrower and final peak position reaches to only $571 \mathrm{~nm}$, namely $26 \mathrm{~nm}$ higher than non-crosslinked one. FWHM of the signal of the films shows a linear increase with respect to FA content under all pressures (Fig. 4c). The viscosity of the medium is dramatically increased by crosslinking. The particle domains prefer to stay in aggregated state because the crosslinking points may hinder their diffusion into individual particles. Similarly, the shift in peak position increases as FA content increases (Fig. 4d). At low FA content, the peak position is close to the signal of individual Au particles. However, at high concentrations, the signal is close to one of their corresponding clusters probably due to the aforementioned formation of a three-dimensional network by crosslinking agent.

Since the PAAm consists of hydrophilic pendant groups on carbon backbone, the material frequently employed in a diverse application as a hydrogel $[37,38]$. PAAm especially having short chain length has hygroscopic nature, hence their solid form may be dramatically affected by even moisture in the air atmosphere $[39,40]$. Therefore, the optical response of PAAm/Au NP sensor films was investigated in a humid environment. The films were exposed to various relative humidity ( $\mathrm{RH} ; 73,80$, and $87 \%$ ) for half an hour a) Pressure (MPa)

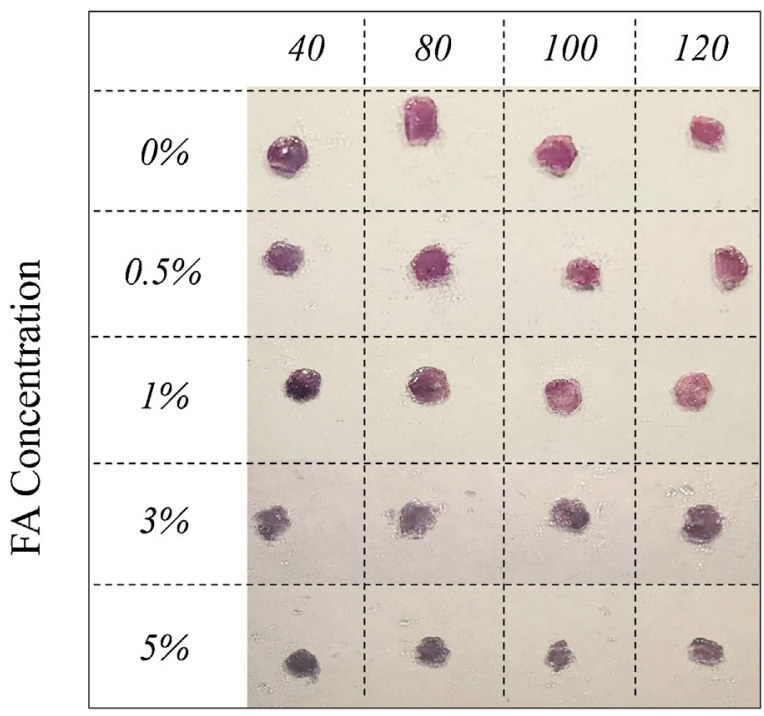

b) $0 M P a$
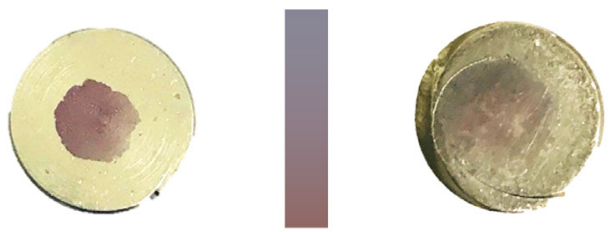

$120 \mathrm{MPa}$

Fig. 6. (a) Photoframe of the films having different FA content under various pressure at $73 \% \mathrm{RH}$. The appearance of the films after compression on (b) smooth (left) and rough (right) surfaces.

and the compression tests were carried out. In Fig. 5a, absorption spectra of the films prepared by non-crosslinked (linear) chains at different $\mathrm{RH}$ conditions for $40 \mathrm{MPa}$ pressure are presented. The spectra remain more or less unchanged under all humidity levels tested. On the other hand, humidity has a strong influence on the crosslinked PAAm matrix and corresponding spectra are shown in Fig. 5b. Compared to the neat counterpart, the signals differentiate dramatically for the films prepared by $5 \%$ FA. The signal falls to $604 \mathrm{~nm}$ for $73 \% \mathrm{RH}$ while it is observed at $566 \mathrm{~nm}$ for $87 \% \mathrm{RH}$. Since the number of remaining aggregates appears more dominant in the crosslinked films, the softening by increasing $\mathrm{RH}$ has a considerable change in absorption compared to the non-crosslinked case. In Fig. 5c and d, FWHM and absorption peak position are given as a function of $\mathrm{RH}$, respectively. Both of them show a linear decrease as the RH increases. It is well established that humidity swells the polymer chains such that the particles find a convenient medium for diffusion. Under pressure, the particle domains can diffuse into the individual particles and the signal shifts toward $540 \mathrm{~nm}$ even for the crosslinked systems.

The crosslinking has a strong influence on the absorption spectrum and as well as the appearance of the films. The photo-frame of the corresponding films is given with respect to the amount of FA in the course of film preparation (column) and applied pressure (row) (Fig. 6a). The color of the film gradually turns into reddish as the pressure increases as each of the rows are followed. The change in color ambiguously observed in the case of a higher degree of crosslinking. The effect of crosslinking at constant pressure on color 
a)
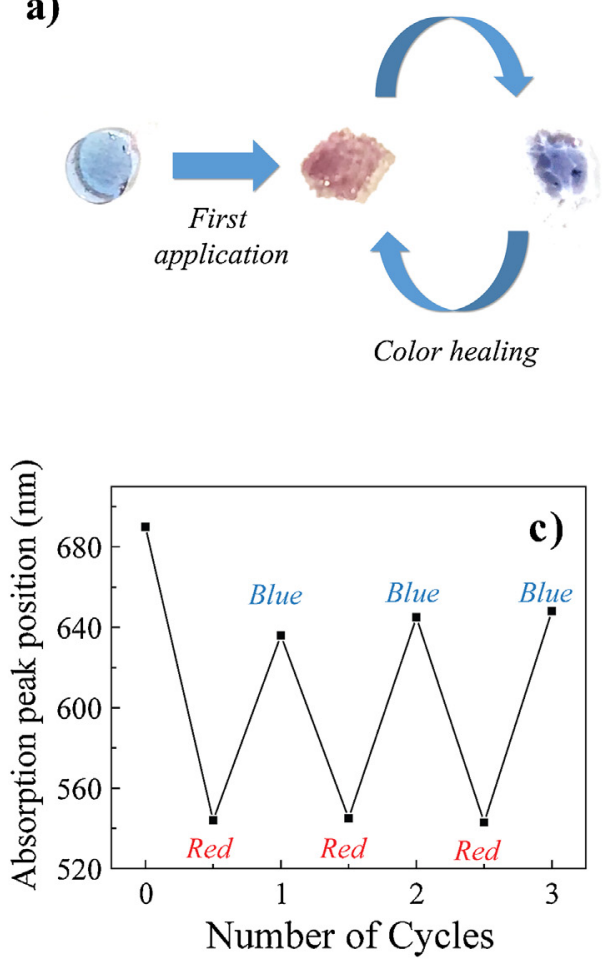
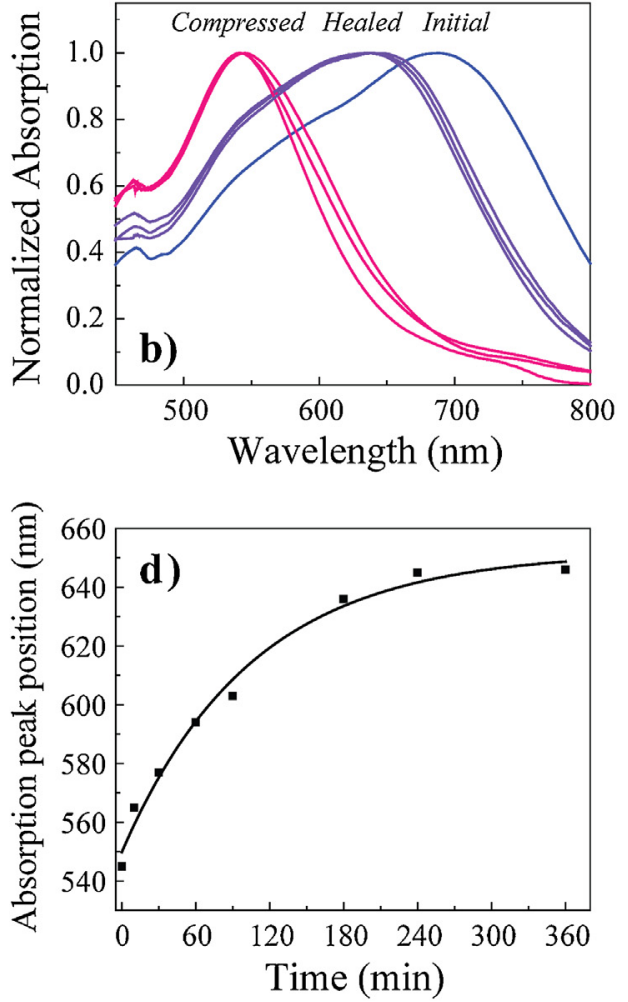

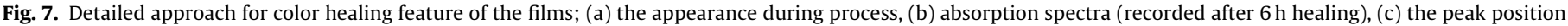
upon color healing cycle, and (d) time evolution of color healing at $95 \% \mathrm{RH}$.

change can be clearly distinguished for each column. In contrast to the effect of pressure, the color turns into bluish as the FA content increases. Note that the change in color with increasing crosslinking is more clearly observed in the case of higher compression. These results may suggest that the films can be employed not only for the measurement of the magnitude of pressure but also for the distribution of the pressure over the material. Two identical films were compressed on smooth and rough surfaces, separately. The photographs of the resulting films are presented in Fig. 6b. The former case leads to smooth and single colored reddish film. It can be claimed that the pressure is homogeneously dispersed over the smooth surface. On the other hand, the latter one resulted as having multiple colors with a gradual transition and seems a mixture of the blue and red, i.e. the pressure is heterogeneously dispersed over the film surface. The change in the color throughout the film may hint about the distribution of applied pressure.

Since the hygroscopic feature of PAAm has a considerable influence on the motion of the Au NPs, the humidity may affect dis/assembly in steady state as much as upon the compression. The film was compressed with $120 \mathrm{MPa}$ and exposed to $95 \% \mathrm{RH}$ for $6 \mathrm{~h}$. The appearance of the film for each stage is presented and corresponding absorption spectra are given in Fig. 7a and b. The initial blue colored film turns into reddish by compression, not surprisingly. Subsequently, the humidity treatment causes color back from reddish to bluish and the absorption signal shifts from 545 to $645 \mathrm{~nm}$. The color healing process may be simply explained by further gelation of the polymers due to excessive humidity. The particles have enough free volume to assemble back that results in bluish colored aggregates. Even though the color cannot be fully healed under humidity, the peak position of the compressed film falls to $545 \mathrm{~nm}$ for each cycle, which may suggest that it is a reversible process (Fig. $7 \mathrm{c}$ ). The time evolution of color healing is presented in Fig. $7 d$. The peak position exponentially increases to $645 \mathrm{~nm}$ and fixed at this wavelength. By providing enough time during the color healing process, the van der Waals interaction between polymer chains and the particles gets more favorable.

\section{Conclusions}

Pressure-sensitive colorimetric PAAm/Au films containing the various degree of crosslinking were fabricated and their sensor properties were investigated in terms of their optical response upon pressure up to $120 \mathrm{MPa}$ under various relative humidity levels. The blue color of the films at the initial state gradually turns into reddish under pressure due to the disassembly of Au NP aggregates, which results as a spectral shift in the absorbance. The shift under certain pressure not only depends on the magnitude of applied force but also internal and external circumstances such as the amount of crosslinking agent in the preparation and humidity of the environment. The characteristic pressure response may enable the use of films as a pressure sensor in different pressure scales. Since the dis/integration of the Au particles is based on the diffusion process in the presence of polymer chains, changing the topology of the polymer chains from linear to crosslinked allows controlling the pressure range where the sensor is active. The usage of formaldehyde retards the colorimetric change under similar pressure, this result suggests that the pressure range required obtaining the same amount of shift increases. The exposure of the deformed films to excessive humidity enables the transforming both in structure and color back to the initial state. Therefore, this healing approach seems a promising way for the reusability of the PAAm/Au films.

\section{Acknowledgements}

The authors gratefully acknowledge funding from The Scientific and Technological Research Council of Turkey (TUBITAK, 1001 117Z331). The authors also thank to the Center for Materials Research of İzmir Institute of Technology. 


\section{References}

[1] N. Sato, S. Shigematsu, H. Morimura, M. Yano, K. Kudou, T. Kamei, K. Machida, Novel surface structure and its fabrication process for MEMS fingerprint sensor, IEEE Trans. Electron Devices 52 (2005) 1026-1032.

[2] C. Metzger, E. Fleisch, J. Meyer, M. Dansachmüller, I. Graz, M. Kaltenbrunner, C. Keplinger, R. Schwödiauer, S. Bauer, Flexible-foam-based capacitive sensor arrays for object detection at low cost, Appl. Phys. Lett. 92 (2008), 013506.

[3] H.-K. Kim, S. Lee, K.-S. Yun, Capacitive tactile sensor array for touch screen application, Sens. Actuators A Phys. 165 (2011) 2-7.

[4] B. Zhu, Z. Niu, H. Wang, W.R. Leow, H. Wang, Y. Li, L. Zheng, J. Wei, F. Huo, X. Chen, Microstructured graphene arrays for highly sensitive flexible tactile sensors, Small 10 (2014) 3625-3631.

[5] C. Pang, G.-Y. Lee, T.-i. Kim, S.M. Kim, H.N. Kim, S.-H. Ahn, K.-Y. Suh, A flexible and highly sensitive strain-gauge sensor using reversible interlocking of nanofibres, Nat. Mater. 11 (2012) 795.

[6] S. Jung, J.H. Kim, J. Kim, S. Choi, J. Lee, I. Park, T. Hyeon, D.H. Kim, Reverse-micelle-induced porous pressure-sensitive rubber for wearable human-machine interfaces, Adv. Mater. 26 (2014) 4825-4830.

[7] C.L. Choong, M.B. Shim, B.S. Lee, S. Jeon, D.S. Ko, T.H. Kang, J. Bae, S.H. Lee, K.E Byun, J. Im, Highly stretchable resistive pressure sensors using a conductive elastomeric composite on a micropyramid array, Adv. Mater. 26 (2014) 3451-3458.

[8] M. Dragoman, V. Ciobanu, S. Shree, D. Dragoman, T. Braniste, S. Raevschi, A Dinescu, A. Sarua, Y.K. Mishra, N. Pugno, Sensing up to $40 \mathrm{~atm}$ Using Pressure-Sensitive Aero-GaN, Phys. Status Solidi Rapid Res. Lett. (2019), 1900012.

[9] G. Schwartz, B.C.-K. Tee, J. Mei, A.L. Appleton, D.H. Kim, H. Wang, Z. Bao, Flexible polymer transistors with high pressure sensitivity for application in electronic skin and health monitoring, Nat. Commun. 4 (2013) 1859.

[10] S. Yao, Y. Zhu, Wearable multifunctional sensors using printed stretchable conductors made of silver nanowires, Nanoscale 6 (2014) 2345-2352.

[11] S.C. Mannsfeld, B.C. Tee, R.M. Stoltenberg, C.V.H. Chen, S. Barman, B.V. Muir, A.N. Sokolov, C. Reese, Z. Bao, Highly sensitive flexible pressure sensors with microstructured rubber dielectric layers, Nat. Mater. 9 (2010) 859.

[12] C. Dagdeviren, Y. Su, P. Joe, R. Yona, Y. Liu, Y.-S. Kim, Y. Huang, A.R. Damadoran, J. Xia, L.W. Martin, Conformable amplified lead zirconate titanate sensors with enhanced piezoelectric response for cutaneous pressure monitoring, Nat. Commun. 5 (2014) 4496.

[13] J. Chun, K.Y. Lee, C.Y. Kang, M.W. Kim, S.W. Kim, J.M. Baik, Embossed hollow hemisphere-based piezoelectric nanogenerator and highly responsive pressure sensor, Adv. Funct. Mater. 24 (2014) 2038-2043.

[14] X. Jin, M. Götz, S. Wille, Y.K. Mishra, R. Adelung, C.J.A.M. Zollfrank, A novel concept for self-reporting materials: stress sensitive photoluminescence in ZnO tetrapod filled elastomers, Communication 25 (2013) 1342-1347.

[15] S. Shree, M. Schulz-Senft, N.H. Alsleben, Y.K. Mishra, A. Staubitz, R. Adelung, interfaces, Light, force, and heat: a multi-stimuli composite that reveals its violent past, ACS Appl. Mater. Interfaces 9 (2017) 38000-38007.

[16] A. Vanderkooy, Y. Chen, F. Gonzaga, M.A. Brook, Silica shell/gold core nanoparticles: correlating shell thickness with the plasmonic red shift upon aggregation, ACS Appl. Mater. Interfaces 3 (2011) 3942-3947.

[17] N. Jiang, X. Zhuo, J. Wang, Active plasmonics: principles, structures, and applications, Chem. Rev. 118 (2017) 3054-3099.

[18] Y. Zhao, L. Cao, J. Ouyang, M. Wang, K. Wang, X.-H. Xia, Reversible plasmonic probe sensitive for $\mathrm{pH}$ in micro/nanospaces based on i-motif-modulated morpholino-gold nanoparticle assembly, Anal. Chem. 85 (2012) 1053-1057.

[19] C. Wang, Y. Du, Q. Wu, S. Xuan, J. Zhou, J. Song, F. Shao, H. Duan, Stimuli-responsive plasmonic core-satellite assemblies: i-motif DNA linker enabled intracellular pH sensing, Chem. Commun. 49 (2013) 5739-5741.

[20] Z. Sun, W. Ni, Z. Yang, X. Kou, L. Li, J. Wang, pH-controlled reversible assembly and disassembly of gold nanorods, Small 4 (2008) 1287-1292.

[21] X.-Y. Liu, F. Cheng, Y. Liu, W.-G. Li, Y. Chen, H. Pan, H.-J. Liu, Thermoresponsive gold nanoparticles with adjustable lower critical solution temperature as colorimetric sensors for temperature, $\mathrm{pH}$ and salt concentration, J. Mater. Chem. 20 (2010) 278-284

[22] M. Tagliazucchi, M.G. Blaber, G.C. Schatz, E.A. Weiss, I. Szleifer, Optical properties of responsive hybrid Au@ polymer nanoparticles, ACS Nano 6 (2012) 8397-8406.

[23] S.E. Lee, Q. Chen, R. Bhat, S. Petkiewicz, J.M. Smith, V.E. Ferry, A.L. Correia, A.P. Alivisatos, M.J. Bissell, Reversible aptamer-Au plasmon rulers for secreted single molecules, Nano Lett. 15 (2015) 4564-4570.
[24] X. Han, Y. Liu, Y. Yin, Colorimetric stress memory sensor based on disassembly of gold nanoparticle chains, Nano Lett. 14 (2014) 2466-2470.

[25] A. Rankin, S. McGarry, A flexible pressure sensitive colour changing device using plasmonic nanoparticles, Nanotechnology 26 (2015), 075502.

[26] G. Topcu, T. Guner, E. İnci, M.M. Demir, Flexible, Colorimetric, and Self-healable Pressure Sensors: A Smart Phone-assisted Detection. Unpublished Work, 2019.

[27] D.P. Bakalik, D.J.J. Kowalski, The reaction of polyacrylamide, formaldehyde, and sodium bisulfite, J. Polym. Sci. Part A: Polym. Chem. 25 (1987) 433-436

[28] D.W. Fong, D.J. Kowalski, An investigation of the crosslinking of polyacrylamide with formaldehyde using 13C nuclear magnetic resonance spectroscopy, J. Polym. Sci. Part A: Polym. Chem. 31 (1993) 1625-1627.

[29] G.B. Marandi, K. Esfandiari, F. Biranvand, M. Babapour, S. Sadeh, G. Mahdavinia, pH sensitivity and swelling behavior of partially hydrolyzed formaldehyde-crosslinked poly (acrylamide) superabsorbent hydrogels, J. Appl. Polym. Sci. 109 (2008) 1083-1092.

[30] V.S.K. Chakravadhanula, Y.K. Mishra, V.G. Kotnur, D.K. Avasthi, T. Strunskus, V. Zaporotchenko, D. Fink, L. Kienle, F.J.Bjon. Faupel, Microstructural and plasmonic modifications in Ag-TiO2 and Au-TiO2 nanocomposites through ion beam irradiation, Beilstein J. Nanotechnol. 5 (2014) 1419-1431.

[31] V. Juvé, M.F. Cardinal, A. Lombardi, A. Crut, P. Maioli, J. Pérez-Juste, L.M. Liz-Marzán, N. Del Fatti, F. Vallée, Size-dependent surface plasmon resonance broadening in nonspherical nanoparticles: single gold nanorods, Nano Lett. 13 (2013) 2234-2240.

[32] T. Klar, M. Perner, S. Grosse, G. Von Plessen, W. Spirkl, J. Feldmann, Surface-plasmon resonances in single metallic nanoparticles, Phys. Rev. Lett. 80 (1998) 4249.

[33] C. Sönnichsen, T. Franzl, T. Wilk, G. von Plessen, J. Feldmann, O. Wilson, P. Mulvaney, Drastic reduction of plasmon damping in gold nanorods, Phys. Rev. Lett. 88 (2002), 077402

[34] U. Kreibig, M. Vollmer, Theoretical Considerations, Springer, 1995.

[35] G. Wegner, M.M. Demir, M. Faatz, K. Gorna, R. Munoz-Espi, B. Guillemet, F. Gröhn, Polymers and inorganics: a happy marriage? Macromol. Res. 15 (2007) 95-99.

[36] M.M. Demir, P. Castignolles, Ü. Akbey, G. Wegner, In-situ bulk polymerization of dilute particle/MMA dispersions, Macromolecules 40 (2007) 4190-4198.

[37] A.K. Denisin, B.L. Pruitt, Interfaces, tuning the range of polyacrylamide gel stiffness for mechanobiology applications, ACS Appl. Mater. Interfaces 8 (2016) 21893-21902.

[38] S. Magdeldin, S. Enany, Y. Yoshida, B. Xu, Y. Zhang, Z. Zureena, I. Lokamani, E. Yaoita, T. Yamamoto, Basics and recent advances of two dimensional-polyacrylamide gel electrophoresis, Clin. Proteomics 11 (2014) 16.

[39] M. Naguib, T. Saito, S. Lai, M.S. Rager, T. Aytug, M.P. Paranthaman, M.-Q. Zhao, Y. Gogotsi, Ti 3 C 2 T x (MXene)-polyacrylamide nanocomposite films, RSC Adv. 6 (2016) 72069-72073.

[40] K.E. Lee, I. Khan, N. Morad, T.T. Teng, B.T. Poh, Thermal behavior and morphological properties of novel magnesium salt-polyacrylamide composite polymers, Polym. Compos. 32 (2011) 1515-1522.

\section{Biographies}

Gokhan Topcu received his BSc in Chemistry (2013) and MSc in Organic Chemistry (2015) from the Marmara University, respectively. He has been carrying out his doctoral research on advanced polymeric composites and nanomaterials at Department of Materials Science and Engineering in Izmir Institute of Technology since 2015. His current researches are on the development of mechanical sensors by using plasmonic and photonic nanostructures in polymeric systems.

Mustafa M. Demir is Professor at the Izmir Institute of Technology, Department of Materials Science and Engineering. He coordinates an interdisciplinary group working on synthesis and nanotechnologies on soft matter. His research activity is focused on the development of polymeric optical nanocomposites for advanced applications, electrospinning, and functional systems. He has been awarded the "Young researcher" Prize of Turkish Academy of Sciences in 2013. His details can be found at: http://www.demirlab.iyte.edu.tr. 